\title{
Les dimensions spatiales du maintien de l'ordre. Introduction
}

Lucie Bony, Muriel Froment-Meurice et Marion Lecoquierre

\section{OpenEdition}

1 Journals

Édition électronique

URL : https://journals.openedition.org/cdg/7553

DOI : $10.4000 /$ cdg. 7553

ISSN : 2107-7266

Éditeur

UMR 245 - CESSMA

\section{Référence électronique}

Lucie Bony, Muriel Froment-Meurice et Marion Lecoquierre, "Les dimensions spatiales du maintien de l'ordre. Introduction », Carnets de géographes [En ligne], 15 | 2021, mis en ligne le 30 avril 2021, consulté le 27 mai 2021. URL : http://journals.openedition.org/cdg/7553 ; DOI : https://doi.org/ $10.4000 /$ cdg.7553

Ce document a été généré automatiquement le 27 mai 2021.

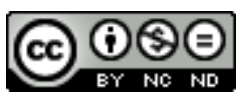

La revue Carnets de géographes est mise à disposition selon les termes de la Licence Creative Commons Attribution - Pas d'Utilisation Commerciale - Pas de Modification 4.0 International. 


\title{
Les dimensions spatiales $\mathrm{du}$ maintien de l'ordre. Introduction
}

\author{
Lucie Bony, Muriel Froment-Meurice et Marion Lecoquierre
}

1 Ce numéro consacré aux dimensions spatiales du maintien de l'ordre s'est construit au cours d'une période fortement marquée par des événements médiatisés, relançant des débats sur les pratiques policières et notamment la relation police-citoyen'ne ${ }^{1}$. Dans ce contexte, le cadre légal et la doctrine du maintien de l'ordre ont fait l'objet de concertations et de différents projets de réformes en France. Le Ministère de l'intérieur a par exemple publié le Schéma national du maintien de l'ordre (SNMO) en septembre $2020^{2}$, largement focalisé sur des situations de manifestations, ainsi que le Livre blanc de la sécurité intérieure en novembre 2020 qui a été suivi du «Beauvau de la sécurité » en $2021^{3}$. La même année, le projet de loi "Sécurité globale ${ }^{4}$ a été aussi fortement débattu, donnant lieu à de nombreuses manifestations d'opposition. Plusieurs articles de ce dossier thématique des Carnets de géographes analysent certaines des grandes tendances abordées dans ces différents documents institutionnels: une attention accrue pour l'anticipation des risques (SNMO, p. 4, p.17), l'utilisation des principes de la "prévention situationnelle » (SNMO, p. 17), la multiplication des acteurices intervenant dans le maintien de l'ordre, notamment d'acteurices privées (Livre blanc, p. 48), ou le développement des capacités technologiques (Livre blanc, p. 264).

2 Si certains articles abordent des questions liées aux doctrines et aux pratiques policières, ce dossier prend toutefois du recul par rapport à cette actualité centrée sur la gestion des foules manifestantes et la dénonciation de violences policières systémiques. Il propose en effet une définition large de ce qu'est le maintien de l'ordre, en s'appuyant notamment sur la notion de «policing ». De fait il envisage une pluralité d'acteurices au-delà du corps policier et il interroge la manière dont les rapports de pouvoir contribuent à (re)produire l'ordre public dans différents contextes, de l'espace manifestant aux jardins partagés, en France mais aussi à l'étranger.

3 L'intérêt partagé par l'ensemble des auteurices pour la dimension spatiale du maintien de l'ordre, étudiés sous différents angles disciplinaires constitue la ligne directrice de ce dossier qui s'inscrit donc dans la continuité d'autres travaux sur le sujet (voir par 
exemple Choplin, Redon, 2014; Herbert 1996, 1997). Cette introduction reviendra d'abord sur les différentes acceptations de l'expression "maintien de l'ordre »: la définition adoptée permet d'envisager les conflits et les collaborations entre différents groupes d'acteurices et met en lumière la circulation et l'hybridation de leurs pratiques. Nous montrerons ensuite comment les articles du dossier abordent les représentations des espaces à policer et les différentes modalités de rapports à l'espace des agentees en charge du maintien de l'ordre. Il ressort enfin du corpus d'articles une prévalence du cadre analytique foucaldien pour aborder la dimension disciplinaire et gouvernementale des dispositifs de contrôle.

\section{Du maintien de l'ordre au « policing »}

On peut distinguer différents usages de l'expression «maintien de l'ordre » en France. Dans les usages policiers et le langage médiatique, le maintien de l'ordre renvoie au " contrôle des foules manifestantes et protestataires" (Jobert, Favre, 2020). Dans une seconde approche, plus large mais toujours relativement restrictive, le maintien de l'ordre peut être défini à partir du champ de compétence de la "police d'ordre ", tel que le définit Dominique Monjardet (1996) à savoir non seulement la gestion des manifestations mais aussi le renseignement intérieur et le contrôle des frontières. Dans cette perspective, le maintien de l'ordre vise à assurer l'encadrement du public, la garde du territoire national, et il dépend de l'État central.

5 Ce dossier envisage le maintien de l'ordre dans une troisième perspective, encore plus large en s'appuyant sur la « distinction cruciale entre police (le policier et la police) et policing (l'activité de police)» des études anglophones (Brodeur, 2003 : 344 ; voir aussi, par exemple, Johnston, 1999: 176). C'est dans cette perspective que nous avons envisagé le maintien de l'ordre dans ce numéro : il recouvre l'ensemble des pratiques visant à préserver l'ordre public, entendu comme la "sécurité, [la] salubrité, [et la] tranquillité » dans l'espace public (Ricci, 2014 : 357). Au sein de l'organisation policière française, ces missions relèvent traditionnellement (mais pas exclusivement, nous le verrons) de la compétence de la "police de sécurité publique» (Monjardet, 1996) ladite « police de proximité » ou les polices municipales - qui se concentre sur l'échelle locale. La circulation de formes de " community policing " participe également de plus en plus à cette "mise en ordre locale » en associant des habitant-e's au travail de la police (Pichonnaz, 2017).

\section{Plural policing et non-police policing}

6 L'approche que nous avons souhaité proposer avec ce dossier repose ainsi sur une définition englobante du maintien de l'ordre, non comme une activité réservée au corps policier, mais comme un ensemble d'activités assurées par différents corps professionnels et par des compagnies privées ou des habitant·es. Le maintien de l'ordre est ainsi à entendre comme un processus de mise en ordre aussi bien matériel que symbolique $^{5}$ de la société. Nous avons souhaité mettre en lumière les acteurices qui contribuent à le (re)produire quotidiennement et les rapports de pouvoir asymétriques qu'iels entretiennent, en analysant plus particulièrement la dimension spatiale de cette activité. En ce sens, il s'agit d'interroger l'ensemble des dispositifs qui visent à limiter ce qu'on appelle couramment les "troubles à l'ordre public", les "petits désordres 
quotidiens » ('disorder') (Skogan, 1990) ou "les incivilités » (Roché, 1996). «L'ordre » dont il est question dans ce dossier n'est donc pas seulement celui des grandes manifestations ou des lieux d'exception mais un ordre plus quotidien qui n'en est pas moins contraignant ou violent.

Cette perspective traduit le développement des travaux autour du "plural policing " (voir par exemple Jones, Newburn, 2006 ; Crawford, 2008; Rogers, 2018) et prend acte de la multiplication de types d'acteurices impliquée's dans le domaine de la sécurité. Ces travaux montrent que l'État ne représente plus qu'un acteur parmi d'autres et que le maintien de l'ordre repose sur des acteurices public.ques à différents échelons territoriaux décentralisés mais aussi des acteurices privé.es fonctionnant en réseau (Loader 2000 ; Jones, Newburn 2006). Ainsi, les articles réunis dans le dossier portent en premier lieu sur les forces de police et les instances relevant de ministères régaliens français (armée, douanes, administration pénitentiaire notamment) montrant que celles-ci ont été et restent les acteurs centraux du maintien de l'ordre. Mais certains articles portent aussi sur différents cas d'étude internationaux, et sur les agents de sécurité privés, les syndicats et les organisations militantes, ou encore les acteurices impliqué.es dans l'aménagement urbain, dans le prolongement d'autres travaux francophones qui ont mis en lumière le travail quotidien des médiateurices sociauxles (Ben Mrad, 2004), des correspondantes de nuit (Benec'h-Le Roux, Maillard, 2011 ; Maillard, 2013), des agentee's dans les espaces de transport ferroviaire (Bonnet, 2008, Castagnino, 2016), des agente's de sécurité privée dans les centres commerciaux (Ocqueteau, 1993) ou encore des groupements spécifiques aux ensembles de logements sociaux (Malochet, 2017).

8 Au-delà de cette pluralisation des acteurices en charge d'assurer le maintien de l'ordre et des mesures de contrôle formel, les articles de ce dossier envisagent aussi la participation d'une multiplicité d'acteurices non professionnelles au maintien de l'ordre local : certain'e-s habitante's parfois organisée's en associations (dans le cadre de jardins partagés, de groupements de quartier) ou des citoyen'ne's assurant le service d'ordre dans les partis politiques. C'est donc toute la diversité des acteurices qui contribuent à maintenir l'ordre dans un espace donné que nous avons souhaité mettre en lumière. Cette diversification et cette hybridation des modèles de policing (Jobard et Maillard, 2015), qui peut qui peut faire écho à des situations historiques de déploiement de milices, de comités de vigilance ou de groupes d'autodéfense (Fourchard, 2018), est telle qu'elle peut conduire à parler de "'policing' beyond the police » (Crawford, 2008 : 147) et même de "non-police policing» (Devroe, Terpstra, 2015). Ces formes d'implication de non-professionnelle's dans le maintien de l'ordre local contribuent à reproduire des rapports de pouvoir structurant ces communautés. C'est ainsi que les pratiques de certain'e's habitante's sont considérées par les institutions compétentes comme plus légitimes que d'autres (Morange, Didier, 2006) et que la mise en œuvre de dispositifs «partenariaux » vient renforcer leur pouvoir d'appropriation de l'espace au détriment d'autres usages définis comme « indésirables».

\section{La judiciarisation du maintien de l'ordre}

Au-delà de la diversification des acteurs impliqués dans le maintien de l'ordre, les articles du dossier portent également un regard original sur l'implication des 
institutions judiciaires, mettant en évidence divers phénomènes relevant de la « judiciarisation du maintien de l'ordre ».

Ils illustrent d'abord la généralisation d'une gestion pénale des problèmes sociaux, et s'inscrivent ainsi à la suite de plusieurs recherches qui montrent la façon dont le droit est mobilisé par les pouvoirs publics pour gérer les « désordres en public » (Chesnay et al., 2014 ; Ranaivo, 2018 ; Poncela, 2010). Dans le dossier, Aurélien Restelli appréhende la judiciarisation de la gestion des foules manifestantes à travers l'exemple des «interdictions préventives» de manifester. Celles-ci prennent diverses formes, administratives ou pénale, et trouvent leur origine aussi bien dans la gestion des supporters ultras de football ou des militants altermondialistes lors des sommets du G8, que dans certaines mesures portées par la loi relative à l'état d'urgence. Qu'il s'agisse de placements en garde à vue avant la manifestation ou d'interdiction de paraître en lieu et date de futurs rassemblements collectifs, la logique est la même : il s'agit de mettre à l'écart des individus et de limiter les potentiels désordres qu'ils pourraient générer. C'est via la mobilité contrainte, l'exclusion et finalement la neutralisation que l'ordre est maintenu. Dans le cadre des manifestations, le recours à ces mesures d'éloignement s'inscrit dans des dynamiques politiques plus larges : les interpellations et déferrements sont d'une part considérés comme des indicateurs de l'activité policière et de son efficacité (la "politique du chiffre») (Filleule, Jobard, 2016); l'interpellation est aussi un instrument de communication qui permet de renforcer un discours sur la dangerosité, la violence et la déviance des protestataires, décrédibilisant par là même le bien-fondé d'un mouvement social (Lippens, 2016 : 74). De plus en plus, les magistrates - juges ou procureur.es - bien qu'éloignées du terrain, jouent un rôleclé dans le maintien de l'ordre.

Sous un autre angle, le dossier donne aussi à voir le rôle des acteurices pénitentiaires comme acteurices du maintien de l'ordre. Ces fonctionnaires dépendent en France du Ministère de la Justice et constituent l'une des forces nationales de sécurité (invitées à défiler le 14 juillet aux côtés des militaires et policiers depuis 2016). L'article de Bénédicte Michalon (et al.), qui évoque notamment l'accueil réservé aux chercheures en milieu pénitentiaire, donne à voir une institution de moins en moins "totale " (Goffman, 1968), au sein de laquelle le pouvoir des surveillantes se reconfigure au contact des intervenantes extérieures de plus en plus diverses (chercheures mais aussi enseignant·es, médecins, acteurices associatif·ves, etc.). Le livre de Marie Morelle, Yaoundé Carcérale. Géographie d'une ville et de sa prison, dont rend compte Franck Ollivon, apporte un autre éclairage sur le "gouvernement de la prison", à partir de la monographie d'une prison camerounaise. Au-delà de l'analyse de l'ordre interne à l'institution carcérale, Marie Morelle interroge plus largement le rôle de la prison dans le maintien de l'ordre à Yaoundé à travers la notion de "continuum carcéral ». Un tel décentrement du regard, depuis l'institution carcérale vers la ville, est aussi opéré par la thèse de Franck Ollivon. Consacrée au placement sous surveillance électronique en France, cette recherche étudie notamment le travail des agente's des services pénitentiaires d'insertion et de probation (SPIP) chargés de réaliser la surveillance et l'accompagnement social des placés; son travail pose la question de l'empreinte carcérale au-delà des murs des prisons et invite à considérer la pluralité des configurations du maintien de l'ordre au-delà de la stricte analyse des modalités de punition et de surveillance. 


\section{Hybridation des pratiques et circulation des modèles}

12 Le "plural policing», les "dispositifs partenariaux", les "échanges de bonnes pratiques » qu'étudient les auteurices de ce dossier mettent en lumière la circulation des doctrines et des méthodes entre différentes acteurices du maintien de l'ordre. Alex Mahoudeau évoque dans son compte-rendu de l'ouvrage de Mathieu Rigouste La domination policière la circulation « endocoloniale » des modèles de maintien de l'ordre, c'est-à-dire l'importation en métropole de techniques, pratiques mais aussi de matériel (les lanceurs de balles non-perforantes par exemple) mis en place afin de contrôler des populations colonisées. Si l'ouvrage étudie le cas de la France, d'autres auteurs ont mis en évidence le même type de processus par exemple au sein de l'empire britannique, avec la circulation d'agentees et de modèles du maintien de l'ordre entre l'Irlande, la Palestine et le reste de l'empire (Sinclair, 2006; Sinclair, Williams, 2007). Cette circulation des pratiques n'est en effet pas nouvelle (Jobard, 2008). Dans le dossier, Clément Monseigne conclut ainsi son article sur une circulation non pas seulement des pratiques mais aussi des hommes, avec les réformes de la force publique durant la révolution et la dissolution des Gardes-françaises, incorporées d'abord dans la garde nationale soldée puis dans l'armée.

D'autres travaux insistent sur l'hybridation des pratiques de différents corps professionnels : les travaux de Laurent Lopez (2009) identifient par exemple dès la fin du xix ${ }^{\text {ème }}$ une "militarisation de la police» comme une "policiarisation de la gendarmerie nationale». Dans le dossier, les travaux d'Arthur oldra montrent comment les opérations Sentinelle conduisent à une policiarisation des forces militaires qui est différemment appréciée par les agent-e's sur le terrain. L'article d'Andrea Kretschmann donne à voir des modalités d'entrainement des forces de police similaires à celles des armées engagées dans des conflits asymétriques et des interventions de guérilla urbaine, avec des simulations effectuées dans des villes artificielles; elle confirme ainsi l'intersection croissante entre espaces militaires et espaces urbains, rejoignant l'analyse faite par Stephen Graham (2010), et met en évidence la transformation des doctrines et du rapport à l'espace des forces de police, notamment sous la pression des politiques antiterroristes, mais aussi de l'utilisation massive de technologies de surveillance.

\section{Rapport à l'espace des acteurices en charge du maintien de l'ordre}

Les articles du dossier donnent également à voir le rapport à l'espace des acteurices en charge du maintien de l'ordre. Celui-ci est d'abord appréhendé sous l'angle de leur affectation géographique, dessinant ainsi des secteurs d'intervention ou des zones de compétence, définies de manière institutionnelle. D'autres articles adoptent une démarche plus microgéographique et analysent le placement dans l'espace des corps des forces de l'ordre. Il apparaît dans plusieurs articles que les zones frontalières et les quartiers populaires font l'objet d'attentions particulières et constituent des archétypes d'espaces à policer. Enfin, ce rapport à l'espace des acteurices en charge du maintien de l'ordre est interrogé sous l'angle des connaissances de terrain qu'ils acquièrent dans leurs formations professionnelles ou in situ. 


\section{Une approche micro de la répartition des forces de l'ordre dans l'espace}

Une approche élémentaire de la dimension spatiale du maintien de l'ordre consiste à s'interroger sur la distribution (fixe ou mobile) des forces de l'ordre dans l'espace.

À l'échelle nationale, cette problématique relève de la géographie de l'État et ouvre des réflexions relatives à l'organisation administrative de la police et de la gendarmerie nationales : l'enjeu est alors de s'interroger sur le maillage du territoire, la place de ces services publics dans les zones considérées comme « sensibles » ou dans les campagnes, les relations État central-collectivités locales, etc. (Mouhanna, 2007; Erzen, 2007). Appréhendée à l'échelle plus locale, cette problématique rejoint celle de la " territorialisation de l'action publique », et pose la question de la coopération localisée entre acteurices impliqué.es dans le maintien de l'ordre (Germain, 2013).

Peu d'articles du dossier s'inscrivent dans cette lecture institutionnelle de l'espace des forces de l'ordre, si ce n'est celui que Jonas Campion consacre à l'histoire de la collaboration policière à la frontière franco-belge. Il y articule une analyse des réformes et accords diplomatiques visant à dépasser la frontière nationale (réseau transmanche Cross Channel Intelligence Cooperation, accords de Tournai, instauration d'un Centre de Coopération policière et douanière) et une étude des modalités concrètes de l'exercice quotidien du maintien de l'ordre. Il dévoile ainsi les leviers et les obstacles à la construction d'une « Europe de la sécurité » (Roché, 2004).

8 Les articles abordent davantage la répartition spatiale des forces de l'ordre à l'échelle micro, dans le placement de leurs corps. Ainsi, Clément Monseigne analyse l'articulation entre " policing fixe » (à partir des postes de garde) ou mobile (à travers les patrouilles). Il analyse la présence des soldats de la Garde française dans l'espace public de l'Ancien Régime comme une mise en visibilité des rapports de force politique entre la royauté et la commune de Paris : «la présence des Gardes-françaises à Paris n'était pas seulement destinée à contenir les foules turbulentes, mais [...] elle représentait également un message adressé aux élites contestataires ». Un autre exemple nous est donné par la thèse d'Arthur Oldra qui est consacrée aux «jeux de places et de placements" des militaires en opération Vigipirate. Elle montre que les places occupées dans l'espace public urbain dépendent de facteurs variés, dont les normes d'usages de l'espace et leur actualisation par les interactions avec les passants. La place occupée est plus généralement révélatrice des représentations que les acteurs en coprésence ont les uns des autres, et de la légitimité et de l'illégitimité qu'ils s'accordent mutuellement. L'article d'Aïcha Bourad et Fanny Parent étudie les meetings politiques et donne également à voir l'importance du placement et de la visibilité des agents chargés du maintien de l'ordre, articulé aux conditions de circulation et de placement du public. Les pratiques diffèrent fortement selon les partis politiques considérés, opposant par exemple un service d'ordre très visible, en rangs serrés, au Rassemblement National alors qu'il est beaucoup plus discret et diffus dans des meetings du Parti socialiste ou du Modem. Les autrices interprètent ces différences comme étant révélatrices de visions du monde et de l'ordre public différentes, étroitement liées aux positions et idées défendues par chaque parti. Ces quelques exemples révèlent que le maintien de l'ordre est un travail de corps à corps (nous revenons plus bas sur les corps contrôlés). L'analyse de la localisation des agents ou des patrouilles dans l'espace, et de leur mise en visibilité, s'avère heuristique et riche en 
significations pour aborder les modalités d'exercice du pouvoir de l'institution qu'ils incarnent.

\section{Deux archétypes d'espaces à policer : la frontière internationale et le quartier populaire}

Il est des espaces qui focalisent l'attention des forces de l'ordre, et dans lesquels elles sont concentrées. Deux exemples nous en sont donnés dans le dossier. Le premier concerne les espaces frontaliers : ils sont en effet considérés comme étant caractérisés par des problématiques spécifiques liées à la mobilité des délinquants et des criminels, aux trafics et fraudes entre pays, et aux questions migratoires.

Les deux articles traitant de la frontière l'appréhendent non comme une simple limite linéaire entre deux territoires mais comme une zone bien plus large : elle constitue un "bassin de criminalité " transnational pour les policiers du Centre de coopération policière et douanière franco-belge étudiés par Jonas Campion; elle s'étend au Calaisis et même au-delà à travers les stratégies de dispersion des personnes migrantes étudiées par Camille Guenebeaud. Les deux articles traitant de cet espace donnent aussi à voir sa particularité d'un point de vue institutionnel parce qu'il combine sécurité intérieure et extérieure et met en contact des institutions d'appartenance nationale différente. Léopoldine Manac'h, dans son compte-rendu de l'ouvrage du collectif BABELS «La police des migrants. Filtrer, disperser, harceler», revient également sur «l'épaississement de la frontière » et la constitution de frontières internes aux États par les policiers eux-mêmes. Ces articles montrent que les différents services de police ou de gendarmerie impliqués sur ces espaces frontaliers en ont des conceptions divergentes. La frontière franco-belge, étudiée par Jonas Campion, est «traitée par les services centraux comme une zone de transit au prisme de la grande criminalité, mais vue par les responsables locaux du centre comme une zone de vie appréhendée sous l'angle de la lutte contre l'insécurité quotidienne ». Camille Guenebeaud donne quant à lui à voir un « rapport et un ancrage au territoire différent » selon les services de police engagés à la frontière franco-britannique à Calais. Ces rapports différenciés à l'espace frontaliers et à ses problématiques d'ordre public influent sur les pratiques et les modalités d'application des stratégies policières : à Calais, les Compagnies républicaines de sécurité (CRS) ont par exemple un rapport plus distancié à leur territoire d'intervention que les gendarmes ou la Police aux frontières (PAF), leurs actions sont alors souvent considérées comme étant relativement plus violentes et moins respectueuses des accords implicites passés par les autorités locales avec les associations.

21 Ces rapports différenciés au territoire et aux populations ont déjà été documentés par des recherches menées sur d'autres terrains. Dominique Monjardet (1996) montre par exemple que les forces d'intervention mobiles (comme les CRS) ont un rapport de circonstance avec le territoire alors que la police judiciaire jongle entre son ressort judiciaire et les spatialités de la criminalité. La police urbaine nécessite quant à elle une plus grande adaptation au terrain ainsi qu'une meilleure compréhension des spécificités locales: Marie Morelle (2017) distingue par exemple différents types de rapport au territoire, qualifié d'« occupation » pour une BST (Brigade spécialisée de terrain), et d'« investissement » pour une BAC (Brigade anti-criminalité) locale. 
Notre corpus d'articles nous montre également que les quartiers populaires focalisent l'attention des forces de l'ordre. Ce ciblage est ancien, comme le révèle Clément Monseigne à partir d'une étude détaillée des trajets effectués par les patrouilles des Gardes-françaises : ceux-ci se concentraient en effet dans les quartiers populaires de Paris, perçus par le pourvoir royal comme des foyers de violence et d'agitation (Farge, 1979), les classes laborieuses étant considérées comme dangereuses (Chevalier, 1958). Dans ces quartiers, les Gardes-françaises étaient non seulement plus nombreuses, mais elles y passaient plus de temps, tout en parcourant des distances moins importantes qu'ailleurs, l'enjeu étant non seulement d'opérer des fouilles minutieuses mais aussi de se montrer et de jouer un rôle dissuasif (voire préventif). Les analyses de Clément Monseigne ne rendent pas compte de violence ou de heurts entre population et forces de l'ordre lors de ces patrouilles (ce qui n'implique pas qu'elles aient été inexistantes). Or on sait qu'à l'époque contemporaine, cette même focalisation de la police sur les quartiers populaires est source de tensions et de violences récurrentes. Les recherches récentes sur le sujet décrivent ainsi le durcissement des politiques sécuritaires dans les quartiers populaires et la dégradation des relations entre habitants et agente-s en charge du maintien de l'ordre (Mucchielli, 2020). L'enquête de terrain de Andrea Kretschmann montre que les villes artificielles créées en Irlande du Nord, en France et en Angleterre pour l'entrainement des forces de police comprennent toutes des quartiers populaires, considérés comme un environnement nécessairement criminogène.

Si aucun de ces articles ne pousse l'analyse jusque-là, on peut s'interroger sur l'existence d'inégalités voire de discriminations territoriales dans l'exercice du maintien de l'ordre. Dans le dossier, Félix Tréguer évoque les discriminations issues de l'adoption de certaines technologies de surveillance, et notamment des logiciels de police préventive: des recherches mettent en effet en garde sur le fonctionnement même de ces logiciels, fondés sur des modèles statistiques nourris de données biaisées, qui tendraient à renforcer un maintien de l'ordre centré sur certaines zones considérées a priori comme criminogènes (Barrett, 2017 ; Brayne, 2017 ; Brantingham, 2018 ; O'Donnell, 2019 ; Richardson et al., 2019). Il souligne ainsi l'intérêt de prendre en considération l'espace dans les analyses des discriminations. Il a en effet été montré par ailleurs que les contrevenants rencontrés dans les quartiers populaires ont un risque plus élevé d'être arrêtés, indépendamment de facteurs tels que le type de crime, les rapports de race ou le comportement du contrevenant (Smith, 1986). De tels « effets de contexte " ont également été repéré dans la répression judiciaire, tant en lien avec le lieu de jugement (Mariette et al. 1995; Cahu, 2017) qu'avec celui d'origine des justiciables (Wooldredge, 2007 ; Shook et Goodkind, 2009).

\section{Formation et apprentissage des pratiques de maintien de l'ordre : distance et proximité du terrain d'intervention}

Plusieurs articles du dossier mettent en lumière les modalités d'acquisition des techniques de maintien de l'ordre. Cette connaissance semble reposer d'une part sur des formations permettant de préparer les forces de l'ordre au déploiement sur le terrain, et d'autre part sur une immersion directe dans les espaces qu'elles ont à policer. 
laquelle il s'est directement confronté à la formation des agents de sécurité en Inde. L'obligation des formations imposées par les États se heurte aux logiques mercantiles des compagnies privées qui les assurent: lorsqu'elles ne sont tout simplement pas inexistantes, ces formations se limitent à l'apprentissage du maintien du corps, de la politesse (que l'auteur associe à de la servilité dans un rapport de classe) et de l'apparence. Ces formations se concentrent sur l'intériorisation de règles de conformation sociale, permettant aux gardiens de performer leur rôle une fois sur le terrain. d'exercice du maintien de l'ordre, c'est également sur le terrain que ces agent·e·s acquièrent un savoir-faire qui est aussi un savoir "être-là » (pour reprendre Arthur Oldra). La familiarisation avec l'environnement d'intervention peut en effet passer par une immersion en son sein : celle-ci permet de maitriser l'organisation spatiale du secteur, de connaître la vie des habitants, de développer de l'interconnaissance, d'obtenir des renseignements, d'être informé de la délinquance locale, etc. C'est par exemple dans cette perspective qu'une réforme de 1854 impose aux policiers d'habiter les quartiers auxquels ils sont affectés : ainsi, « les policiers se transforment en "bornes sensorielles" (Deluermoz, 2012: 106) car ils développent des dispositifs phénoménologiques d'alerte, nourris par leur visibilité et leur disponibilité, et par la connaissance intime qu'ils ont du quartier et de sa population » (Moreau De Bellaing, 2013).

28 Cette immersion est souvent valorisée, comme le décrit ce gendarme : « Le temps de présence dans l'affectation, la connaissance de la circonscription et de la population, le contact avec les habitants sont des critères très importants pour le gendarme dans son travail au quotidien. Cela représente un gain de temps pour limiter le temps d'intervention, une sécurité dans l'approche d'un délinquant local et dans sa recherche $\mathrm{du}$ renseignement sur le terrain " (Choplin, Redon, 2014). Cette valorisation d'une connaissance fine du terrain est également perceptible dans les différents systèmes visant à inclure les résidents et à valoriser la question du «lien social» - figure rassurante du contrôle social - pour «pacifier» le quartier. La " pluralisation » des acteurices du maintien de l'ordre (Johnston, 2003) peut ainsi amener une délégation de certaines prérogatives à des acteurices privé·es, considéré·es comme ayant une bonne 
connaissance du terrain et une pratique intime, quotidienne de ses enjeux. Naoko Tokumitsu montre ainsi comment la police japonaise s'appuie sur des comités de quartiers et des résidents âgés pour la «mise en ordre » du quartier et pour contrôler les comportements considérés comme déviants. C'est aussi dans cette logique qu'est régulièrement débattue en France la pertinence du retour d'une " police de proximité " et la pratique de l'îlotage. Mais cette immersion est perçue comme ayant des limites, une trop grande proximité avec la population pouvant à l'inverse générer des risques de compromission voire de corruption. Ainsi, gendarmes et policiers sont amenés à régulièrement changer d'affectation (Choplin, Redon, 2014). Les changements réguliers de lieu de casernement pratiqués durant l'Ancien Régime, évoqués par clément Monseigne visaient déjà à « distendre les liens qu[e les Gardes-françaises] pouvaient tisser avec la population d'un quartier ».

\section{Des dispositifs de contrôle pour discipliner les corps}

Les contributions du dossier rendent également compte du caractère pluridisciplinaire du champ de recherche relatif au maintien de l'ordre. Les articles des historiens, et leurs bibliographies, témoignent de l'ampleur du tournant spatial qui touche la discipline, au-delà de la seule historiographie française de la police. A cela s'ajoutent les contributions de sociologues et de politistes, les apports des géographes étant finalement relativement minoritaires dans le dossier. Si les contributeurices viennent d'horizons disciplinaires variés, nombre d'entre euxelles mobilisent les travaux de Michel Foucault dans leurs analyses du maintien de l'ordre. Cela n'est pas surprenant, la police étant une notion cardinale de l'œuvre du philosophe (Denis, 2013). D'après Vincent Denis (ibid.), la préoccupation des historiens pour l'inscription de l'action policière dans l'espace résulte aussi de l'influence de Michel Foucault. Au vu des contributions reçues, on peut étendre cette hypothèse à une partie des travaux de ce dossier.

\section{Des objets foucaldiens ? Un intérêt pour les dispositifs de contrôle et de surveillance}

Les dispositifs de contrôle et de surveillance ont été particulièrement étudiés dans les travaux anglophones qui se singularisent même comme un champ spécifique - les 'surveillance studies'. Certains vont jusqu'à affirmer que «Foucault (1977) (bien qu'écrivant sur les siècles passés) est le grand-père des surveillance studies contemporaines $^{6} »($ Marx, 2015, p. 2, tl.). Dans cette lignée, les recherches ont porté plus particulièrement sur des idéaux-types comme le panoptique ('panopticon'), la société disciplinaire ('disciplinary society') ou "la société de sécurité maximale» ('maximum security society') (ibid). Dans le champ de la géographie française, les analyses sur le panoptique, la prison (Milhaud, 2009) et plus globalement les institutions disciplinaires, ou la vidéosurveillance (Lecat-Deschamps, 2014) sont les exemples archétypaux de cet héritage foucaldien.

Différents dispositifs sont ainsi étudiés par les auteurices de ce dossier qui partagent une attention particulière pour la manière dont le contrôle de l'espace participe des processus de maintien de l'ordre. Aurélien Restelli analyse la maitrise de l'espace manifestant par les forces de l'ordre. Il met en lumière le «service préliminaire » qui 
consiste à aménager l'espace manifestant dans l'objectif de le «vider " (fermer les chantiers et les stations Vélib', faire retirer les poubelles à verre et les véhicules automobiles, faire protéger les abribus et les écrans publicitaires, et prévenir les commerçants des risques de dégradations). Aïcha Bourad et Fanny Parent analysent " la dimension spatiale comme paramètre central des modes de contrôle du public par les organisateurs du meeting" dans le cadre de la campagne pour l'élection présidentielle de 2012. Elles mettent en lumière la manière dont l'organisation de l'espace contribue à structurer les groupes mais aussi les "visions du monde » propres à chaque parti politique envisagé. Dans la lignée d'autres travaux sur les utopies urbanistiques comme Michelinville (Védrine, 2014) ou les jardins urbains (Glatron, Granchamp, 2018), Romane Joly et Vincent Lebrou mettent en lumière la fonction disciplinaire des jardins partagés et comment l'«ordonnancement de l'espace " (rationalisation de l'organisation de l'espace, uniformisation des équipements) est « au service du maintien de l'ordre ».

Ces velléités de contrôler des populations par le contrôle de la production de l'espace et l'agencement de ses éléments matériels s'appuient désormais de plus en plus sur des technologies qui renouvellent les modalités de surveillance des populations. Des chercheures ont ainsi étudié les nouvelles technologies de contrôle et de surveillance : vidéosurveillance (Heilmann, 2008; Mucchielli, 2018), bracelet électronique avec ou sans géolocalisation (Razac, 2010; Allaria, 2014; Ollivon, 2018), drones (Chamayou, 2013), y compris dans des objets du quotidien comme les toilettes publiques (FromentMeurice, 2016). Dans ce numéro Aurélien Restelli aborde la question de l'équipement des policiers encadrant les manifestations, Arthur Oldra celui des patrouilles des opérations Sentinelle, tandis que Naoko Tokumitsu détaille le cas de «super lampadaires connectés » implantés dans des quartiers péri-urbains de Tokyo. Iels interrogent la façon dont ces dispositifs transforment l'exercice du maintien de l'ordre et plus particulièrement ses dimensions spatiales. Le recours à la vidéosurveillance automatisée (VSA) et aux outils Big Data ont notamment un effet direct sur la ville et le maintien de l'ordre, tout comme les modèles de "Smart» et de "Safe City " qui promeuvent une administration urbaine assistée par ordinateur, à l'aide d'algorithmes exploitant et croisant des bases de données massives pour faciliter l'organisation des flux et des services mais aussi la prise de décision. Cela conduit à une " datafication » (Egbert, 2019), ou « mise en données », croissante du maintien de l'ordre.

33 Ces technologies peuvent directement influencer les pratiques des acteurices du maintien de l'ordre: leur dimension préventive est de plus en plus transformée en visée prédictive (voir par exemple Jankowski 2015 ; Kaufmann et al., 2019) ce qui peut se traduire par exemple dans une modification du parcours des patrouilles ou des lieux d'intervention.

Le développement de ces technologies de la surveillance participe également de la diversification des acteurices impliquées dans le maintien de l'ordre avec le développement d'un lucratif marché de la sécurité. Dans ce dossier, Félix Tréguer met ainsi en lumière le rôle du secteur privé et plus particulièrement des $\mathrm{GAFAM}^{7}$ dans le déploiement de ces nouvelles technologies, et interroge les enjeux économiques comme politiques de ces modalités de contrôle, et les dangers qu'elles représentent pour les libertés publiques. 


\section{Des concepts foucaldiens : un héritage à bas bruit ?} Rabinow, 1984) plus qu'à celle de l'injonction et de la répression (Foucault, 2004). Cette gouvernementalité transparaît dans le cas des jardins partagés étudiés par Romane Joly et Vincent Lebrou qui mettent en lumière "les mécanismes de surveillance et de contrôle non-coercitifs qui transparaissent dans l'injonction à l'alignement des conduites selon un modèle prescrit ». Aïcha Bourad et Fanny Parent distinguent deux types de gouvernementalité dans les meetings électoraux qu'elles étudient. La première forme de "gouvernementalité " repose sur la mise en scène de la distance entre militant·e's et candidatees, sur la personnalisation des candidate's et la théatralisation du meeting électoral. La deuxième forme de gouvernementalité repose au contraire sur la mise en scène de la proximité entre militant-e's et candidate's et repose au contraire sur la dépersonnalisation des candidate's et la valorisation de l'interconnaissance. Peu des articles interrogent les formes de subjectivation de ces dispositifs depuis le point de vue des groupes ciblés ; c'est plutôt la manière dont ces dispositifs sont appropriés par les acteurices du maintien de l'ordre qui constitue le cœur de ce dossier. Damien Carrière montre ainsi que le contrôle des corps passe aussi par le contrôle des corps des contrôleurs eux-mêmes, en analysant comment les gardiens de sécurité doivent apprendre à "performer » leur rôle. Andrea Kretschmann mobilise également une référence foucaldienne en analysant les espaces de formation des polices européennes comme des "hétérotopies ", c'est-à-dire "un autre espace réel, aussi parfait, aussi méticuleux, aussi bien arrangé que le nôtre est désordonné, mal agencé et brouillon » (Foucault, 1984 : 759, cité par l'auteure). Dans l'article d'Andrea Kretschmann le terme s'applique aux villes artificielles utilisées comme espaces d'entrainement par les forces de police en Irlande du Nord, France, Allemagne et Angleterre. Ces espaces permettent de reproduire des situations perçues comme typiques des opérations de maintien de 
l'ordre, et leur analyse permet à l'autrice d'identifier des "géographies assaillantes " dont les quartiers populaires sont une cible récurrente dans tous les contextes nationaux envisagés. La mise en œuvre dans ces espaces de formation de «scénarios apocalyptiques » contribue à structurer tant les expériences que les représentations des policièrees.

\section{Identifier et contrôler des « indésirables »}

Au-delà de ce cadre théorique fortement inspiré des travaux de Michel Foucault, les articles du dossier soulignent combien ces techniques ont pour but de contrôler des corps et des circulations. Leur analyse permet de fait aux auteurices d'identifier différentes "figures d'indésirables" (Froment-Meurice, 2016) selon les contextes envisagés. Dans les jardins partagés étudiés par Romane Joly et Vincent Lebrou les conduites qu'il s'agit de normaliser sont celles des jeunes hommes racisés des quartiers populaires reproduisant ainsi des « représentations plus généralement partagées qui imputent aux populations juvéniles, souvent des hommes racisés, érigées en 'nouvelle classe dangereuse' (Chevalier, 1958), les 'problèmes des banlieues' (Tissot, 2007)». Les auteurices ont une approche critique de la mise en œuvre des jardins partagés car ils peuvent devenir "instrument de relégation des 'indésirables' ». Comme dans de nombreux travaux déjà publiés sur le sujet (Harcourt, 2006; Garnier, 2002), Naoko Tokumitsu souligne combien le recours à la prévention situationnelle permet, en mobilisant des registres techniques et urbanistiques, de contrôler en fait certains individus et groupes sociaux construits comme "dangereux ». Elle relève que ce type de politique reproduit et renforce d'autres dynamiques d'exclusion vis-à-vis des personnes sans-domicile fixe notamment (voir aussi Terrolle, 2004), ou des "jeunes » vus comme déviants (Tabet, 1999). Cette construction de l'indésirabilité transparait aussi dans les articles des historiens : Jonas Campion montre notamment comment la question du maintien de l'ordre à la frontière franco-belge durant l'entre-deux guerres se cristallise autour de la circulation des Tziganes et des étrangers. A une époque plus contemporaine, l'article de Camille Guenebeaud aborde de front les violences subies par les personnes migrantes à Calais. Au-delà de la diversité des figures d'indésirables que révèle l'analyse de ces dispositifs, ils permettent aussi d'interroger des rapports de pouvoir plus structurels (de race, de classe, de genre, d'âge) qui inscrivent résolument ces travaux dans les approches critiques (Morange, Calbérac, 2012).

Au-delà de la dimension spatiale du contrôle des groupes et individus définis comme indésirables, les auteurices mettent également en lumière la diversité des modalités de contrôle : «l'enfermement » « l'éloignement », mais aussi « la dispersion », « la mise en circulation", "l'invisibilisation». Camille Guenebeaud analyse par exemple des « dispositifs spatiaux de contrôle frontalier » et les «stratégies de mise en mouvement forcées » qui «enferment les personnes dans des situations d'errance». Ces analyses rejoignent celles de de Bénédicte Michalon (2013) sur d'autres "dispositifs spatiaux " de rétention des étrangers qui reposent sur « deux fondements : privation de liberté de mouvement et (re)mise (forcée) en mouvement ", la mise circulation étant finalement au service de l'enfermement. Léopoldine Manac'h rend compte du travail de l'équipe Babels pour documenter «l'omniprésence des forces de police dans la répression des migrations et ses conséquences en termes de violences ", avec des méthodes reposant sur trois piliers: "filtrer, disperser, harceler». Dans son compte-rendu de La Domination Policière. Une violence industrielle, Alex Mahoudeau montre comment les 
"dispositifs de répression employés par la police reposent sur la séparation, le quadrillage, et l'envahissement de quartiers » populaires et ciblent une population racisée.

\section{Questions ouvertes, pistes à explorer : méthodologie de recherche, espaces ruraux, régimes autoritaires}

Ce numéro thématique a permis de poursuivre, d'approfondir et d'élargir la réflexion entamée par de précédents dossiers de revue (Choplin, Redon, 2014; Morelle, Tadié, 2011) sur la dimension spatiale du maintien de l'ordre. Il confirme de grandes tendances (circulation des modèles, technologisation aigüe, intérêt pour la prédiction, pluralisation des acteurs, ...), mais met également en évidence quelques angles mort de la recherche, notamment en géographie, ou, tout du moins, des questions peu ou pas traitées dans le présent numéro méritant d'être explorées dans de futures contributions.

\section{Quelles spécificités des enquêtes auprès des acteurices du maintien de l'ordre?}

41 Des retours d'expérience nous sont donnés dans les deux articles de la rubrique «Carnets de terrain ». B. Michalon, T. Bruslé, O.Clochard, M. Darley, O. Milhaud et M. Morelle évoquent ainsi les difficultés rencontrées pour entrer sur leurs terrains respectifs, les obstacles administratifs érigés par les institutions, notamment l'Administration pénitentiaire en France ( 0 . Milhaud) et de son équivalent au Cameroun (M. Morelle). L'accès physique aux lieux d'enquête, l'observation directe et la circulation des chercheurs au sein des espaces fermés est ainsi limité, et contrôlé. L'accès aux centres de rétention pour personnes migrantes étudiés par $\mathrm{B}$. Michalon en Roumanie et M. Darley en Allemagne, tout comme aux zones de rétention administratives étudiées par 0 . Clochard demandent également des négociations et des " examens d'entrée ", parfois soldés par un échec. Les auteurs notent cependant un «mouvement d'ouverture relative des institutions de réclusion» qui n'est pas observable dans le cas des acteurs privés gérant les «labour camps » étudiés au Qatar par T. Bruslé et en Espagne par Djemila Zeneidi. Ces obstacles limitant l'accès au terrain donnent lieu à des adaptions et tactiques de contournement, notamment par l'intermédiaire d'associations et d'ONG.

Au-delà de l'accès physique aux lieux d'enquête, la recherche pose également la question classique de la place du chercheur vis-à-vis des enquêtés, qui passe également par la place du corps et de l'engagement corporel : dans le cadre de sa thèse sur le plan Vigipirate, Arthur Oldra a ainsi réalisé une enquête en immersion, en tant que réserviste de l'Armée de Terre, et analyse la manière dont les corps, civils et militaires, sont "placés» dans l'espace. Damien Carrière évoque lui aussi dans son article les méthodes adoptées dans la recherche pour avoir accès à certains enquêtés, en l'occurrence les gardiens de sécurité en Inde. Afin de mieux comprendre leur parcours, il a ainsi suivi des séances de formation destinées à ces gardiens, se confrontant aux changements des contextes politiques présidant à ces formations - souvent annulées. Ces formes d'observation participante interrogent la dimension corporelle de la recherche, ici avec l'apprentissage commun de postures martiales; l'auteur insiste 
également sur l'altérité du chercheur sur des terrains étrangers, où le genre, la couleur de peau, l'origine géographique ou l'accent influencent à la fois la position du chercheur et la perception qu'en ont les enquêtés.

Les questions éthiques que soulèvent ce rapport aux enquêtée's (la manière dont cela influence les méthodes de recherche, leurs résultats ou leur diffusion) ne sont pas explicitement abordées dans ce dossier. Au-delà de ces trois articles centrés sur des enjeux méthodologiques, ils donnent à voir la diversité des méthodes mobilisables pour traiter le sujet (recherches en archives, entretiens, observations, analyse de littératures grise, etc.) et invitent à s'interroger sur les conditions de réalisation des recherches menées sur de tels "terrains sensibles" (Bouillon et al., 2006). En effet, certaines institutions peuvent avoir une tendance au secret et sont par là même plus ou moins ouvertes aux chercheures et à la publicisation de leurs pratiques. D'où des difficultés d'accès aux archives (Berlière, 2009, 2015) ou aux acteurices du maintien de l'ordre pour les observer ou les interroger (Monjardet, 2005). Quelles " ruses et arts de faire " (de Certeau, 1990) sont mobilisés par les chercheurees? Quelles difficultés pose l'observation (directe comme participante) qui peut entraîner des frictions avec les institutions tutélaires, les agentees en charge du maintien de l'ordre comme avec certains groupes policés?

Il serait également intéressant de questionner les modalités de restitution des recherches et les conséquences que peut avoir la publication auprès des personnes enquêtées ou des institutions : cette étape particulièrement sensible est peu traitée ici. La crainte de compromettre un accès ultérieur au terrain a-t-elle un effet sur ce que les chercheur'e's s'autorisent à diffuser? Comment, et à qui les chercheure's rendent des comptes? Alex Mahoudeau rappelle combien la production du savoir est fonction du point d'énonciation où se situent les auteurices dans l'espace social et du rapport qu'iels entretiennent avec les personnes enquêtées (Bensa, Fassin, 2008; Naudier, Simonnet, 2011). Son compte-rendu de l'ouvrage La domination policière souligne que Matthieu Rigouste écrit aux côtés de, avec et pour les personnes qui subissent quotidiennement la violence policière. C'est le même point de vue situé qui conduit le collectif Babels à multiplier les livres de petit format accessibles à un large public pour documenter et analyser la «crise des politiques d'hospitalité » (Akoka, 2017). Ainsi, certaines auteurices cherchent à contribuer plus largement, au-delà des cercles académiques, au débat politique sur le maintien de l'ordre, voire directement à la vie politique. C'est également le cas de Félix Tréguer, membre de La Quadrature du Net, association qui revendique des victoires dans la lutte contre le déploiement de certaines technologies de surveillance. L'entretien que nous avons réalisé avec lui propose une réflexion plus explicite sur le sujet: il y aborde de front sa double appartenance de chercheur et de militant, évoquant une posture de "recherche adversariale » à la fois féconde et contraignante. L'engagement politique et les postures critiques posent-elles de manière plus aiguë les questions déjà évoquées d'une possible restriction d'accès au terrain ou à certaines sources?

\section{Des espaces et des thématiques à explorer?}

45 La majorité des contributions du dossier portent leur attention sur les espaces urbains. Les espaces ruraux semblent ainsi échapper au regard des enquêtes sur le maintien de l'ordre. La contribution d'Andrea Kretschmann montre néanmoins que la police française s'entraîne dans un milieu pensé comme une Zone A Défendre (ZAD) construite 
sur le modèle de Notre-Dame des Landes. Mais au-delà des évènement ponctuels ou exceptionnels (manifestations, occupations) qu'en est-il du maintien de l'ordre au quotidien en zone rurale? De même, les articles du dossier se concentrent majoritairement sur les espaces publics (à l'exception peut-être des meetings étudiés par Fanny Parent et Aïcha Bourad) où les problématiques de maintien de l'ordre sont les plus évidentes, redoublant en quelque sorte la focalisation des institutions et des médias sur ce type d'espace. Doit-on en conclure que les espaces domestiques ne relèvent pas de ce champ d'action? Les lettres de cachet qui permettaient aux familles de faire enfermer les "perturbateurs de l'ordre intime" au xviiie siècle (Farge, Foucault, 1982), mais aussi les pratiques contemporaines d'hospitalisation sous contraintes invitent au contraire à réfléchir à la façon dont les problématiques d'ordre public peuvent s'immiscer dans la sphère privée.

Par ailleurs, les textes reçus traitent presque exclusivement du maintien de l'ordre dans des démocraties, et principalement des démocraties européennes. L'analyse des spatialités du maintien de l'ordre au sein de régimes illibéraux ou autoritaires aurait ainsi pu apporter un autre éclairage, notamment vis-à-vis de la conception de l'espace public ou des répertoires de protestation et de répression. L'analyse de ces contextes semble d'autant plus importante dans une période marquée par la normalisation de mesures limitant les droits des citoyens, entre état d'urgence et état d'exception.

La mise au jour de similarités ou de différences dans les pratiques de maintien de l'ordre selon les contextes incite à caractériser différentes politiques de maintien de l'ordre. Par exemple, pour les manifestations, «certaines techniques [...] sont communes aux [...] polices européennes, comme l'accompagnement du cortège sur ses flancs, le tronçonnement (les "nasses» vues en 2016) et d'autres encore " (Filleule, Jobard, 2018: 27) ; inversement les spécificités françaises de ce maintien de l'ordre (affrontement, conflictualité) et leurs conséquences sont parfois opposées au modèle allemand (désescalade). De telles réflexions sur la circulation à l'échelle internationale d'expériences ou de modèles dans les pratiques policières, judiciaires ou encore militaires du maintien de l'ordre sont peu explorées dans ce dossier. Pour autant, il serait intéressant d'identifier les modalités de diffusion de certains de ces modèles, ainsi que les freins ou les résistances rencontrées. Ces circulations méritent aussi d'être appréhendées dans une perspective économique: comment se structure l'espace du marché de la sécurité ? Si l'on observe une concentration des producteurs d'armes à l'échelle mondiale (Boulanger, 2006), qu'en est-il des autres technologies mobilisées dans le maintien de l'ordre? Dans la lignée d'autres travaux sur la circulation des modèles urbains (Peyroux, Sanjuan, 2016), la dimension comparative et les approches géographiques du transfert des politiques de maintien de l'ordre demeurent une piste féconde pour de futures publications.

\section{BIBLIOGRAPHIE}

AGAMBEN G. (2017), The Omnibus: Homo Sacer, Stanford, Stanford University Press. 
AKOKA K. (2017), « Ce n'est pas une crise des migrants mais une crise des politiques d'hospitalité », Projet, no. 360, vol. 5, pp. 77-83.

ALLARIA C. (2014), « Le placement sous surveillance électronique : espace et visibilité du châtiment virtuel », Champ pénal/Penal field [En ligne], Vol. XI.

BABELS (2019), La police des migrants. Filtrer, disperser, harceler, Coordonné par Sarah Barnier, Sara Casella Colombeau, Camille Gardesse, Camille Guenebeaud \& Stefan Le Courant, Paris, Éditions du Passager clandestine.

BARRETT, L. (2017), « Reasonably Suspicious Algorithms: Predictive Policing at the United States Border », New York University Review of Law \& Social Change, no. 41, vol. 3, pp. 327-366.

BRANTINGHAM, P. (2018), “The logic of data bias and its impact on place-based predictive policing”, Ohio State Journal of Criminal Law, no. 15, vol. 2, pp. 473-486.BRAYNE, S. (2017), "Big Data Surveillance: The Case of Policing", American Sociological Review, no. 82, vol. 5, pp. 977-1008.

DE BACKER M., MELGAÇO L., VARNA G., MENICHELLI F. (dir.) (2016), Order and Conflict in Public Space, Londres, Routledge.

BEN MRAD F., 2004, « La médiation sociale : entre résolution des conflits et sécurisation urbaine », Revue française des affaires sociales, no. 3, pp. 231-248.

BENEC'H-LE ROUX P., DE MAILLARD J. (2011), « Évaluation de l'activité des correspondants de nuit de la Ville de Paris ", Note de synthèse, Convention CESDIP / Ville de Paris n 2010 DPP 22, $24 \mathrm{f}$.

BENIT-GBAFFOU C. (2004), « Nous avons dû prendre la loi entre nos mains ». Pouvoirs publics, politique sécuritaire et mythes de la communauté à Johannesburg », Raisons politiques, no. 15, vol. 3, pp. 53-67.

BENSA A., FASSIN D. (2008), Les politiques de l'enquête. Épreuves ethnographiques, Paris, La Découverte.

BERLIERE J-M. (2001), « Archives de police/historiens policés? », Revue d'histoire moderne \& contemporaine, vol. 5, no. 48-4bis, pp. 57-68.

BERLIERE J-M. (2009), « Archives « interdites », archives « spéciales » ? Quelques réflexions à propos des Archives policières... », Histoire@Politique. Politique, culture, société, no. 8.

BONNET F., 2008, «Les effets pervers de la sécurité. Polices publiques et privées dans une gare et un centre commercial », Sociologie du travail, no. 50, pp. 505-520.

BOUILLON F., FRESIA M., TALLIO V. (dir.) (2006), Terrains sensibles. Expériences actuelles de l'anthropologie, Paris, EHESS.

BRODEUR J.-P., 2003, Les visages de la police. Pratiques et perceptions, Montréal, Les Presses de l'Université de Montréal.

CAHU E., 2017, Géographie de la justice pénale en France : L'équité à l'épreuve des territoires, Thèse de doctorat en géographie, Université de Caen.

CASTAGNINO F. (2016), « Séparer pour mieux surveiller. Spatialité des risques et pratiques de surveillance en Gare du Nord », Flux, no. 103-104, pp. 44-56.

CHAMAYOU G., 2013, Théorie du drone, Paris, La Fabrique.

CHESNAY C., BELLOT C., SYLVESTRE M-È. (2014), « Judiciarisation des personnes itinérantes à Québec : une géographie des pratiques policières répressives au service de la revitalisation », EchoGéo [En ligne], no. 28. 
CHEVALIER L. (1958), Classes laborieuses et classes dangereuses à Paris pendant la première moitié du XIXe siècle, Paris, Plon.

CHOPLIN A., REDON M. (2014), « Gendarmes, policiers : quelles pratiques spatiales ? », EchoGéo [En ligne], 28. DOI : https://doi.org/10.4000/echogeo.13868.

CRAWFORD A. (2008), « Plural policing in the UK: policing beyond the police », in Newburn Tom (dir.), Handbook of Policing, Devon, Willan Publishing, 147-181.

DE CERTEAU M. (1990), L'invention du quotidien t.1 - arts de faire, Paris, Gallimard.

DELUERMOZ Q. (2012), Policiers dans la ville. La construction d'un ordre public à Paris (1854-1914), Paris, Publications de la Sorbonne.

DENIS V. (2013), « L'histoire de la police après Foucault. Un parcours historien », Revue d'histoire moderne et contemporaine, vol. 60, no. 4-4bis, pp. 139-155.

DEVROE E., TERPSTRA J. (2015), « Plural policing in Western Europe, A comparison », European Journal of Policing Studies, vol. 2, no. 3, pp. 235-244.

DREYFUS H., RABINOW P. (1984), Michel Foucault, un parcours philosophique, Paris, Gallimard.

EGBERT Simon, 2019, « Predictive policing and the platformization of police work ", Surveillance and Society, vol. 17, no. 1-2, pp. 83-88.

ERZEN F. (2007), « Du bon usage de l'ancrage territorial ou la vraie tactique du gendarme », Revue historique des armées, no. 248, pp. 20-28.

FARGE A. (1979), «Les théâtres de la violence à Paris au XVIIIe siècle », Annales : Économies, sociétés, civilisations, vol. 34, no. 5, pp. 984-1015.

FARGE A., FOUCAULT M. (1982), Le désordre des familles, Paris, Gallimard.

FASSIN D. (2011), La force de l'ordre. Une anthropologie de la police des quartiers, Paris, Editions Le Seuil.

FILLIEULE O., JOBARD F. (2016), « Un splendide isolement. Les politiques françaises du maintien de l'ordre », La Vie des idées [En ligne]. URL : https://laviedesidees.fr/Un-splendideisolement.html

FOUCAULT M. (1975), Surveiller et punir, Paris Gallimard.

FOUCAULT, M. (1984), « Des espaces autres. Hétérotopies », in Dits et écrits : 1954-1988, t. IV (19801988), Paris, Gallimard, pp. 752-762.

FOUCAULT M. (2004), Sécurité, territoire, population. Cours au collège de France 1977-1978, Paris, Gallimard/Seuil.

FOURCHARD L. (2018), «État de littérature. Le vigilantisme contemporain. Violence et légitimité d'une activité policière bon marché », Critique internationale, no. 78, pp. 169-186.

FROMENT-MEURICE M. (2016), Produire et réguler les espaces publics contemporains. Les politiques de gestion de l'indésirabilité à Paris, Thèse de doctorat en géographie, Université Paris Est Créteil.

GARNIER JP (2002), « Un Espace lndéfendable, L'Aménagement Urbain à L'Heure Sécuritaire », Cidades- Comunidades e Territórios, no. 2, pp. 43-52.

GARO I. (2020), « COVID-19 et capitalisme : le triomphe de la biopolitique ? », Contretemps, [En ligne], https://www.contretemps.eu/coronavirus-capitalisme-biopolitique-foucault-agambenesposito-marxisme/ 
GERMAIN S. (2008), Les politiques locales de sécurité en France et en Italie. Une comparaison des villes de Lyon, Grenoble, Bologne et Modène, Thèse de doctorat de science politique, Université Pierre Mendès-France / Institut d'études politiques de Grenoble.

GERMAIN S. (2013), «Les cadres policiers face à la territorialisation de l'action publique : une institution à la conquête du local », Sciences de la société, vol. 90, pp. 42-57

GLATRON S., GRANCHAMP L. (2018), The Urban Garden City: Shaping the City with Gardens Through History, New York, Springer International Publishing.

GOFMANN E (1968), Asiles. Études sur la condition sociale des malades mentaux, Paris, Editions de Minuit.

GRAHAM S. (2010), Cities under siege. The new military urbanism, Londres, Verso [trad. Villes sous contrôle. La militarisation de l'espace urbain, 2012, Paris, La Découverte].

GRANJON F. (2012), « La critique est-elle indigne de la sociologie ? », Sociologie, no. 1, vol. 3 [En ligne], https://www.cairn.info/revue-sociologie-2012-1-page-75.htm

HARCOURT B. (2006), L'illusion de l'ordre. Incivilités et violences urbaines : tolérance zéro, Paris, Descartes \& Cie.

HERBERT S. (1997), « Territoriality and the police », Professional Geographer, no. 49, vol.1, pp. 86-94.

HERBERT S. (1996), « The normative ordering of police territoriality: Making and marking space with the Los Angeles Police Department ", Annals of the Association of American Geographers, no. 86, vol. 3, pp. 567-582.

HEILMANN E. (2008), « La vidéosurveillance, un mirage technologique et politique » in Mucchielli L., La frénésie sécuritaire, Paris, La Découverte, pp. 113-124.

JANKOWSKI M. (2015), « Big Data : de la police préventive à la police predictive », Revue Défense Nationale, no. 779, vol. 4, pp. 19-21.

JOBARD F. (2008), « La militarisation du maintien de l'ordre, entre sociologie et histoire », Déviance et Société, vol. 32, no. 1, pp. 101-109.

JOBARD F., FAVRE P. (2020), « Maintien de l'ordre », in Fillieule O. (dir.), Dictionnaire des mouvements sociaux, Paris, Presses de Sciences Po, pp. 357-363.

JOBARD F., MAILLARD J. (de) (2015), Sociologie de la police. Politiques, organisations, réformes, Armand Colin, Paris.

JOH E. (2016), The new surveillance discretion: Automated Suspicion, Big Data, and Policing, Harvard Law and Policy Review, no. 10, vol. 1, pp. 15-42.

JOHNSTON L. (1999), « Private Policing in Context », European Journal on Criminal Policy and Research, no. 7, pp. 175-196.

JOHNSTON L. (2003), « From 'pluralisation' to 'the police extended family': Discourses on the governance of community policing in Britain », International Journal of the Sociology of Law, no. 31, vol. 3, pp. 185-204.

JONES, T., NEWBURN, T. (2006), Plural policing, A comparative perspective, Londres, Routledge.

KAUFMANN M., EGBERT S., LEESE M. (2019), « Predictive policing and the politics of patterns », British Journal of Criminology, no. 59, vol. 3, pp 674-692. 
LECAT-DESCHAMPS J-A. (2014), De la biopolitique à la datapolitique. Eléments de philosophie de l'urbain, Thèse de doctorat en urbanisme, aménagement et politiques urbaines, Université Paris-Est Institut d'Urbanisme de Paris.

LOADER I. (2000), "Plural Policing and Democratic Governance”, Social \& Legal Studies, no. 9, vol. 3, pp. 323-345.

LIPPENS B. (2016), Vers une individualisation de la gestion des foules? L'influence des « violences urbaines » et des lanceurs de balles de défense sur le maintien de l'ordre, Mémoire de recherche Master 2 science-politique, Université Paris 1 Panthéon Sorbonne.

LÓPEZ L. (2009), « Ce que fait le maintien de l'ordre aux gendarmes et aux policiers. La force publique sous l'effet d'une interaction professionnelle contrainte (1870-1914) ", Vingtième Siècle. Revue d'histoire, no. 102, vol. 2, pp. 93-104.

MAILLARD (de) J. (2013), « Réguler les espaces publics : le rôle ambivalent des nouveaux métiers ", Métropolitiques [en ligne], https://metropolitiques.eu/Reguler-les-espaces-publicsle.html.

MALOCHET V. (2017), « Contours et positionnement d'une forme hybride de policing résidentiel », Champ pénal/Penal field, vol. XIV, [en ligne], https://journals.openedition.org/ champpenal/9622.

MARIETTE V., LEMERLE S., TIMBART O., BABADJI L. (1995), La physionomie des contentieux selon les tribunaux correctionnels, Paris, Ministère de la justice.

MARX G. T. (2015) «Surveillance Studies », International Encyclopedia of the Social \& Behavioral Sciences, Second Edition, Amsterdam : Elsevier, pp. 733-741.

MICHALON B., 2012, « La mobilité au service de l'enfermement ? », Géographie et cultures, no. 81, [en ligne], http://journals.openedition.org/gc/188.

MILHAUD O. (2009), Séparer et punir. Les prisons françaises mise à distance et punition par l'espace, Thèse de doctorat en géographie, Université Michel de Montaigne Bordeaux 3.

MONJARDET D. (2005), « Gibier de recherche, la police et le projet de connaître », Criminologie, no. 38 , vol. 2, pp. 13-37.

MONJARDET D. (1996), Ce que fait la police. Sociologie de la force publique, Paris, La Découverte. MORANGE M., CALBERAC Y. (2012), « Géographies critiques "à la française" ? ", Carnets de géographes, no. 4, [en ligne], http://www.carnetsdegeographes.org/archives/sommaire_04.php. ffhalshs-01471794f

MORANGE M., DIDIER S. (2006), "Security discourses, community participation and the power structure in Cape Town, 2000-2006”, Urban Forum, no. 17, vol. 4, pp. 353-379.

MOREAU DE BELLAING C. (2013), « Comment la force devient publique », La Vie des idées [en ligne], https://laviedesidees.fr/Comment-la-force-devient-publique.html.

MORELLE M. (2017), « La fabrique de territoires policiers. Des pratiques professionnelles en débat dans une commune francilienne ", Droit et société, vol. 3, no. 97, pp. 469-484.

MORELLE M. (2019), Yaoundé carcérale. Géographie d'une ville et de sa prison, Lyon, Editions ENS.

MORELLE M., TADIÉ J. (2011), « Pratiques de sécurité en ville », Justice spatiale - Spatial Justice, no. 4 [en ligne], https://www.jssj.org/article/pratiques-de-securite-en-ville-introduction/.

MOUHANNA C. (2007), « La répartition des forces de sécurité sur le territoire : des leçons à tirer pour la carte judiciaire », Actualité juridique Pénal, no. 12, pp. 518-520. 
MUCCHIELLI L. (2020), « La dégradation des relations entre les habitants des quartiers pauvres et la police se lit aussi dans les chiffres ", Métropolitiques [en ligne], https://metropolitiques.eu/A-1heure-d-importantes-mobilisations-contre-les.html.

MUCCHIELLI L. (2018), Vous êtes filmés! Enquête sur le bluff de la vidéosurveillance, Paris, Armand Colin.

NAUDIER D., SIMMONET M. (dir.) (2011) Des sociologues sans qualités ? Pratiques de recherche et engagements, Paris, La Découverte.

O'DONNELL R. M. (2019), “Challenging racist predictive policing algorithms under the equal protection clause”, New York University Law Review, no. 94, vol. 3, pp. 544-580.

OCQUETEAU F. (1993), « Les centres commerciaux, cibles d'incivilités et promoteurs de sécurité », Déviance et société, vol. 17, no. 3, pp. 235-260.

OLLIVON F. (2018), La prison chevillée au corps. Pour une approche géographique du placement sous surveillance électronique, Thèse de doctorat en géographie, Université Lyon 3.

PEYROUX E., SANJUAN T. (2016), « Stratégies de villes et « modèles » urbains : approche économique et géopolitique des relations entre villes ", EchoGéo, no. 36, [En ligne], http:// journals.openedition.org/echogeo/14642 ; DOI : https://doi.org/10.4000/echogeo.14642

PICHONNAZ D. (2017), Devenirs policiers. Une socialisation professionnelle en contrastes, Lausanne, Antipodes.

PONCELA P. (2010), « La pénalisation des comportements dans l'espace public », Archives de politique criminelle, no. 32, pp. 1-21.

RAZAC O. (2010), « Le placement sous surveillance électronique mobile (PSEM) : un nouvel espace de la peine ? », Les cahiers de la sécurité, no. 12, pp. 209-215.

RANAIVO A-S. (2018), Sans domicile fixe et droit, Thèse de doctorat en droit, Université Paris 1 Panthéon Sorbonne.

RICCI R., 2014, « Ordre public », in Kada N., Mathieu M. (dir.), Dictionnaire d'administration publique, Presses universitaires de Grenoble, pp. 357-358.

RICHARDSON R., SCHULTZ J. M., \& CRAWFORD K. (2019), “Dirty Data, Bad Predictions: How Civil Rights Violations Impact Police Data, Predictive Policing Systems, and Justice”, New York University Law Review Online, vol. 94, pp. 15-55.

RIGOUSTE M. (2012), La Domination Policière. Une violence industrielle, Paris, La Fabrique.

ROCHE S. (2004), «Vers la démonopolisation des fonctions régaliennes : contractualisation, territorialisation et européanisation de la sécurité intérieure », Revue française de science politique, vol. 54, no. 1, pp. 43-70.

ROCHE S. (1996), La société incivile. Qu'est ce que l'insécurité ?, Paris, Editions du Seuil.

RUUTEERE M., POMMEROLLE M. (2003), « Democratizing security or decentralizing repression? The ambiguities of community policing in Kenya », African Affairs, no. 102, vol. 409, pp. 587-604.

SHOOK J. J., GOODKIND S. A. (2009), « Racial disproportionality in juvenile justice: The interaction of race and geography in pretrial detention for violent and serious offenses ", Race and Social Problems, vol. 1, n4, pp. 257-266.

SINCLAIR Georgina (2006), « 'Get into a Crack Force and earn $£ 20$ a Month and all found...': The Influence of the Palestine Police upon Colonial Policing 1922-1948 ", European Review of History, no. 13 , vol. 1, pp. 49-65. 
SINCLAIR Georgina, WILLIAMS Chris A. (2007), « 'Home and Away': The Cross-Fertilisation between 'Colonial' and 'British' Policing, 1921-85 », The Journal of Imperial and Commonwealth History, no. 35, vol. 2, pp. 221-238.

SKOGAN W. G. (1990), Disorder and decline. Crime and the Spiral of Decay in American Neighbourhoods, Los Angeles, University of California Press.

SMITH D. A. (1986), « The neighborhood context of police behavior », Crime and Justice, vol. 8 , pp. 313-341.

TABET J. (1999), « La résidentialisation du logement social à Paris. Paradoxes et retournement des discours et des pratiques dans les opérations de requalification des grands ensembles », Les annales de la recherche urbaine, no. 83-84, pp. 155-163.

TERROLLE D. (2004), « La ville dissuasive, l'envers de la solidarité avec les SDF », Espaces et Sociétés, no. 1-2, vol.116-177, pp. 143-158.

TISSOT S. (2007), L'État et les quartiers. Genèse d'une catégorie de l'action publique, Paris, Le Seuil.

VEDRINE C. (2014), « Des espaces disciplinaires aux espaces de jouissance : les transformations de Michelinville », Espaces et sociétés, vol. 158, no. 3, pp. 135-149.

WOOLDREDGE J. (2007), « Neighborhood Effects on Felony Sentencing », Journal of Research in Crime and Delinquency, vol. 44, no. 2, pp. 238-263.

\section{NOTES}

1. La répression de différents mouvements sociaux comme la "révolution des parapluies " à Hong Kong en 2014, le mouvement des Gilets jaunes en France en 2018-2019 et l'internationalisation du mouvement «Black Lives Matter » en 2020 ont donné lieu à d'âpres critiques contre des violences policières considérées comme systémiques.

2. Schéma national du maintien de l'ordre, https://www.interieur.gouv.fr/fr/Le-ministre/ Actualites/Schema-national-du-maintien-de-l-ordre, consulté le 12 avril 2021.

3. Livre blanc de la sécurité intérieure, https://www.interieur.gouv.fr/Actualites/L-actu-duMinistere/Livre-blanc-de-la-securite-interieure, consulté le 12 avril 2021.

4. «Proposition de loi pour une sécurité globale préservant les libertés ", https:// www.legifrance.gouv.fr/dossierlegislatif/JORFDOLE000042563668/, consulté le 12 avril 2021.

5. Voir par exemple : De Backer et al., 2016.

6. 'Foucault (1977) although writing about earlier centuries, is the grandfather of contemporary surveillance studies (Marx, 2015: 2).

7. Acronyme désignant les multinationales Google, Apple, Facebook, Amazon, Microsoft.

\section{AUTEURS}

\section{LUCIE BONY}

Chargée de recherche au CNRS, Centre de Recherche sur l'Habitat - UMR 7218 Lavue.

lucie.bony[at]cnrs.fr 


\section{MURIEL FROMENT-MEURICE}

Maitresse de conférences en géographie, Université Paris Ouest Nanterre la Défense, UMR 7218 Lavue,

GT JEDI - Justice Espace Discriminations Inégalités.

muriel.fromentmeurice[at]parisnanterre.fr

\section{MARION LECOQUIERRE}

Docteure en géographie, post-doctorante à l'université d'Helsinki.

marion.lecoquierre[at]gmail.com 